\title{
DETERMINATION OF SEISMOGRAPH CONSTANTS FROM THE CALIBRATION CURVE
}

\author{
By Simón Gershanik and Carlota Gershanik
}

\begin{abstract}
The explicit expression of the long-period WWSSN calibration curve can be obtained solving the differential equations of the seismograph system in terms of $e^{w}$ and identifying the characteristic equation to a product of polynomials of the second degree. The coefficients of these polynomials are the equivalent constants. These and a scale factor can be obtained from the calibration curve. The frequency response of the seismograph system is fully determined by these elements provided that the reduced motor constant and the calibration current are known. The explicit knowledge of the seismograph partial constants is not necessary.

Newton-Raphson's method is generally efficient to obtain the elements from the calibration curve, but it may fail some times. Levenberg's method (slightly modified) and also simple step reduction can be helpful in such cases. The unknowns can be obtained either freely or under the constraint that some of the partial constants have the values assigned to them. The latter may be convenient when the behavior of parts of the system is affected by some trouble.
\end{abstract}

\section{INTRODUCTION}

Without much error, a seismograph may be assumed to be a linear system. Consequently, being $f(t)$ the Earth acceleration, $s(t)$ the function recorded, and representing these functions by the Fourier integrals

$$
s(t)=\frac{1}{2 \pi} \int_{-\infty}^{+\infty} S(\omega) e^{J t \omega t} d \omega ; \quad f(t)=\frac{1}{2 \pi} \int_{-\infty}^{+\infty} F(\omega) e^{j \omega t} d \omega
$$

we may write

$$
S(\omega)=F(\omega) H(\omega) e^{j p(\omega)}
$$

where $H(\omega)$ is the dynamic magnification and $\nu(\omega)$ the phase delay of the system. Expressions for $F(\omega)$ and $\nu(\omega)$ as depending on the partial instrumental constants were given first by Coulomb and Grenet (1935) and later by Hagiwara (1958).

Since the partial constants do not keep their values, and this affects the values of $H(\omega)$ and $\nu(\omega)$, some time ago it was proposed at Lamont Geological Observatory to do a daily calibration signal on the seismograph from which changes in the response can be detected. A step excitation was found to be convenient because it is easily achieved and the Fourier transform of the corresponding response is proportional to $H(\omega) e^{j \nu(\omega)} / j \omega$.

Espinosa et al. (1965) gave a set of curves from which some of the constants can approximately be obtained, and J. Brune, in an Appendix to their paper, showed how to acquire them numerically. Teng and Ben Menahem (1965) tried to obtain $H(\omega)$ by calculating numerically the Fourier transform of the calibration curve. They found that, for high frequencies, the result becomes inaccurate. To improve this method, Mitchell and Landisman (1969) proposed another one where a set of 
parameters introduced in $H(\omega) e^{j(\omega)}$ minimizes the squared difference between the theoretical and the actual calibration curve. The authors pointed out that the method is unable to yield all the constants, and since the coupling factor is small, they assumed it to be null. A variation to this method, based upon explicit expressions for the calibration curve still assuming zero for the coupling factor, has been given by Jarosch and Curtis (1973) who obtained their results applying the Laplace transform to the seismometer equations.

In this paper, we are intending to do a further step in the same sense. We first show that the explicit expressions can be easily obtained by means of the characteristic equation method, and second that, although the partial instrumental constants cannot be obtained separately, it is possible to obtain other parameters from which $H(\omega) e^{j \nu(\omega)}$ is determined without any assumptions. We also refer to the method of obtaining them, indicating how to improve the Newton-Raphson method (which is generally suitable to this end) when it fails to be convergent.

\section{The Seismometer Equations}

After Chakrabarty and Chudhury (1964) or Morencos (1972), the response of an electromagnetic seismograph to a ground acceleration, $f(t)$, is described by the following system of equations

$$
\begin{aligned}
& L_{1} \dot{I}_{1}+q_{1} I_{1}-q_{2} I_{2}=c_{1} \dot{x} \\
& L_{2} \dot{I}_{2}+q_{3} I_{2}-q_{2} I_{1}=c_{2} \dot{s} \\
& \dot{x}+2 \epsilon_{1}{ }^{\prime} \dot{x}+n_{1}{ }^{2}+c_{3} I_{1}=-f \\
& s+2 \epsilon_{2}{ }^{\prime} \dot{s}+n_{2}{ }^{2} s-c_{4} I_{2}=0 \\
& n_{1}=\frac{2 \pi}{T_{1}}, \quad n_{2}=\frac{2 \pi}{T_{2}} .
\end{aligned}
$$

The values $T_{1}, \epsilon_{1}{ }^{\prime}, I_{1}, L_{1}$, correspond to the sensor and are, respectively, the period, the damping coefficient, the circulating current, and the self-inductance. $T_{2}$, $\epsilon_{2}{ }^{\prime}, I_{2}, L_{2}$ are similar values corresponding to the galvanometer; $s$ represents, as before, the ordinates of the record, $x$, the displacement of the oscillating center of the sensor, and $q_{1}, q_{2}, c_{1}, c_{2}, c_{3}$, and $c_{4}$ are constants of the system defined by its electric and electromagnetic characteristics.

Since the case $L_{1} \neq 0, L_{2} \neq 0$ is cumbersome and can be treated in a similar way to the case $L_{1}=0, L_{2}=0$, we shall restrict ourselves, for the sake of simplicity, to the latter. Such assumption can be accepted for the long-period instruments of the WWSSN where the influence of the self-inductances has proved to be relatively small. As was pointed out by Salvill et al. (1962), the same, instead, is not valid for the short-period instruments. A detailed treatment corresponding to these instruments with the method given here is left to a future paper.

Putting $L_{1}=0, L_{2}=0$, the system (1) becomes

$$
\begin{gathered}
x+2 \epsilon_{1} \dot{x}+n_{1}^{2} x-\sigma_{1} \dot{s}=-f \\
\ddot{s}+2 \epsilon_{2} \dot{s}+n_{2}^{2} s-\sigma_{2} \dot{x}=0 .
\end{gathered}
$$

$\sigma_{1}$ and $\sigma_{2}$ are transmission coefficients, their product

$$
\sigma^{2}=\sigma_{1} \sigma_{2}
$$


measures the coupling between the sensor and the galvanometer, and $\epsilon_{1}, \epsilon_{2}$ are their damping coefficients.

The systems (1) and (2) are also valid when the sensor, being of the rotating type, is acted by a moment or when, being of the displacement type, is acted by a force, except for the meaning of $f$ which is different. Taking this into account, let us consider that being at rest, the sensor is acted by a step current $i_{p}$. Then

$$
f=-\frac{G i_{p}}{M}
$$

where $G$ is the reduced motor constant and $M$ the mass of the sensor.

Defining an auxiliary function

$$
y=x-\frac{G i_{p}}{n_{1}^{2} M},
$$

we obtain by replacing it into (2)

$$
\begin{aligned}
& y+2 \epsilon_{1} \dot{y}+n_{1}^{2} y-\sigma_{1} \dot{s}=0 \\
& \dot{s}+2 \epsilon_{2} \dot{s}+n_{2}^{2} s-\sigma_{2} \dot{y}=0
\end{aligned}
$$

and the following initial conditions

$$
t=0 ; \quad y=y_{0}=-\frac{G l_{p}}{n_{1}^{2} M} ; \quad \dot{y}=0 .
$$

$G i_{p} / n_{1}{ }^{2} M$ is the deflection of the sensor caused by $i_{p}$ and equations (3) correspond to the case in which the sensor is free from external actions. Thus, the galvanometer response to the step current is identical to the one resulting after releasing the sensor, and the expression of the calibration curve can be obtained from either equations (3) or (2). Here, we shall obtain it using equations (3).

\section{The Expression of the Calibration Curve}

To solve (3), we assume that

$$
y=A e^{w t} \quad s=B e^{w t}
$$

We then obtain the characteristic equation

$$
\Phi(w)=\left(w^{2}+2 \epsilon_{1} w+n_{1}^{2}\right)\left(w^{2}+2 \epsilon_{2} w+n_{2}^{2}\right)-\sigma^{2} w=0
$$

and

$$
\frac{A}{B}=\frac{w^{2}+2 \epsilon_{2} w+n_{2}^{2}}{\sigma_{2} w} .
$$

To get to the roots of (5), we replace $\Phi(w)$ by the product of the following two quadratic polynomials

$$
\begin{aligned}
& \lambda_{1}(w)=w^{2}+2 a_{1} w+p_{1} \\
& \lambda_{2}(w)=w^{2}+2 a_{2} w+p_{2} .
\end{aligned}
$$


$\lambda_{1}$ and $\lambda_{2}$ contain four constants called by Tobyáš and Hubernova (1969) equivalent constants related to the five partial constants contained in (5) as follows

$$
\begin{aligned}
a_{1}+a_{2} & =\epsilon_{1}+\epsilon_{2} \\
4 a_{1} a_{2}+p_{1}+p_{2} & =4 \epsilon_{1} \epsilon_{2}+n_{1}{ }^{2}+n_{2}{ }^{2}-\sigma^{2} \\
a_{1} p_{2}+a_{2} p_{1} & =\epsilon_{1} n_{2}{ }^{2}+\epsilon_{2} n_{1}{ }^{2} \\
p_{1} p_{2} & =n_{1}{ }^{2} n_{2}{ }^{2} .
\end{aligned}
$$

The roots of (5) are obviously the ones of $\lambda_{1}$ and $\lambda_{2}$, viz.

$$
w_{1,2}=-a \pm \sqrt{a_{1}^{2}-p_{1}} \quad w_{3,4}=-a_{2} \pm \sqrt{{a_{2}^{2}-p_{2}}^{2}}
$$

and the solution of (3) becomes

$$
y=\sum_{1}^{4}{ }_{l} A_{\imath} e^{w_{\imath} t} \quad s=\sum_{1}^{4}{ }_{\imath} B_{\imath} e^{w_{\imath} t}
$$

$A_{\iota}$ and $B_{\iota}$ being determined from the initial conditions and from equations like (6). Assuming that the galvanometer is at rest when $t=0$, we obtain after some algebra

$$
s=\frac{K}{g}\left[e^{-a_{1} t}\left(\cosh b_{1} t+\frac{\kappa_{1}}{b_{1}} \sinh b_{1} t\right)-e^{-a_{2} t}\left(\cosh b_{2} t-\frac{\kappa_{2}}{b_{2}} \sinh b_{2} t\right)\right]
$$

TABLE 1

Cases Taken for Comparison

\begin{tabular}{ccrcc}
\hline Case & $T_{1}$ & $T_{2}$ & $h_{1}$ & $h_{2}$ \\
\hline a & 15.0 & 1000 & 1.0 & 1.0 \\
b & 15.0 & 1000 & 15 & 1.0 \\
$\mathrm{c}$ & 30.0 & 75.0 & 08 & 08 \\
$\mathrm{~d}$ & 30.0 & 75.0 & 1.5 & 60 \\
\hline
\end{tabular}

where

$$
\begin{aligned}
& K=\frac{G i_{p} \sigma_{2}}{M} ; \quad g=\left(a_{1}+\kappa_{1}\right) p_{2}-\left(a_{2}-\kappa_{2}\right) p_{1} \\
& \kappa_{1}=a_{1}+\frac{p_{2}-p_{1}}{2\left(a_{1}-a_{2}\right)} ; \quad \kappa_{2}=-\left(a_{2}+\frac{p_{2}-p_{1}}{2\left(a_{1}-a_{2}\right)}\right) \\
& b_{1}=\sqrt{a_{1}^{2}-p_{1}} ; \quad b_{2}=\sqrt{a_{2}^{2}-p_{2}} .
\end{aligned}
$$

In (8), $b_{1}$ and $b_{2}$ are supposed to be real. Being imaginary, they should be replaced by their modulus and the hyperbolic by the corresponding trigonometric functions. When $b_{1}=0$, the first parenthesis becomes $1+\kappa_{1} t$. Similarly when $b_{2}=0$, the second parenthesis becomes $1-\kappa_{2} t$.

When $\sigma_{1}=0$, expression (8) coincides with the expression obtained by Jarosch and Curtis (1973) and represents the curves given by Espinosa et al. (1965). The values of these curves taken from their maximum onward and the values obtained from (8) for four cases indicated in Table 1, are compared in Table 2. 
The values obtained from (8), putting $K=5.0$, were reduced by multiplying them by a factor $m$ given by

$$
m=\frac{\sum s_{1} s_{2}}{\sum s_{1}{ }^{2}} .
$$

$s_{1}$ is given by $(8)$ and $s_{2}$ is taken from the curves. Both are in millimeters. It appears as though the differences are small.

TABLE 2

Theoretical and Experimental Values Compared

\begin{tabular}{|c|c|c|c|c|c|c|c|c|c|}
\hline \multirow{2}{*}{$t(\mathrm{sec})$} & \multicolumn{2}{|c|}{ Case a } & \multicolumn{2}{|c|}{ Case b } & \multicolumn{2}{|c|}{ Case $\mathrm{c}$} & \multicolumn{3}{|c|}{ Case $\mathrm{d}$} \\
\hline & $s_{1}{ }^{*}$ & $s_{2} \dagger$ & $s_{1}{ }^{*}$ & $s_{2} \dagger$ & $s_{1}^{*}$ & $s_{2} \dagger$ & $t(\mathrm{sec})$ & $s_{1}^{*}$ & $s_{2} \dagger$ \\
\hline & & & & & & & 36 & 85.8 & 874 \\
\hline 22 & 1570 & 1595 & & & 1745 & 178.0 & 40 & 85.5 & 86.5 \\
\hline 24 & 1553 & 1560 & 1467 & 150.0 & 173.0 & 175.0 & 60 & 78.4 & 78.0 \\
\hline 32 & 1347 & 1350 & 1362 & 134.0 & 137.4 & 135.0 & 80 & 69.0 & 68.0 \\
\hline 40 & 106.2 & 1040 & 1124 & 111.0 & 87.3 & 83.0 & 100 & 60.1 & 59.5 \\
\hline 48 & 79.2 & 780 & 86.5 & 850 & 464 & 43.0 & 120 & 52.2 & 51.5 \\
\hline 56 & 570 & 550 & 636 & 640 & 20.0 & 160 & 140 & 454 & 45.0 \\
\hline 64 & 399 & 380 & 453 & 460 & 57 & 40 & 160 & 394 & 390 \\
\hline 72 & 275 & 270 & 31.5 & 32.0 & -07 & -2.0 & 180 & 342 & 35.0 \\
\hline 80 & 186 & 185 & 216 & 225 & -2.7 & -3.5 & 200 & 29.8 & 29.4 \\
\hline 88 & 125 & 125 & 14.6 & 150 & -27 & -30 & 220 & 25.9 & 255 \\
\hline 96 & 8.3 & 80 & 9.7 & 10.2 & -2.0 & -2.3 & 240 & 22.4 & 22.0 \\
\hline 104 & 54 & 5.5 & 6.4 & 65 & -1.2 & -1.5 & 260 & 19.5 & 192 \\
\hline 112 & 36 & 2.8 & 4.2 & 4.5 & -0.6 & -0.8 & 280 & 16.9 & 16.9 \\
\hline 120 & 23 & 18 & 28 & 3.0 & -03 & -04 & 300 & 14.7 & 145 \\
\hline 128 & 15 & 12 & 18 & 2.0 & -0.1 & -0.1 & 320 & 12.8 & 12.5 \\
\hline 136 & 10 & 08 & 1.2 & 12 & -0.1 & -0.1 & 340 & 111 & 11.0 \\
\hline 144 & 06 & 05 & 0.8 & 08 & 0 & -0.1 & 360 & 9.7 & 9.6 \\
\hline 152 & 04 & 0.2 & 05 & 0.6 & & & 420 & 6.4 & 6.2 \\
\hline 160 & 03 & 0.1 & 0.3 & 0.3 & & & 480 & 4.2 & 4.3 \\
\hline 168 & 0.2 & 01 & 0.2 & 02 & & & 540 & 2.8 & 3.0 \\
\hline 176 & 0.1 & 01 & 0.1 & 0.1 & & & 600 & 1.8 & 20 \\
\hline 184 & 0.1 & 01 & 0.1 & 01 & & & 660 & 1.1 & 1.5 \\
\hline$t_{\max } \ddagger$ & 21.5 & 210 & 245 & 23.5 & 22.3 & 22.0 & & 36.4 & 36.0 \\
\hline$t_{0} \S$ & & & & & 707 & 70.0 & & & \\
\hline
\end{tabular}

* Values obtained from equation (8).

$\uparrow$ Values given graphically by Espinosa et al. (1965).

$\ddagger$ Values of $t$ corresponding to a maximum of $s$.

$\S$ Values of $t$ corresponding to $s=0$

\section{Constants Obtained from the Calibration Curve}

The values of $a_{1}, a_{2}, p_{1}, p_{2}$, and $K$ can be obtained from a convenient set of equations (8) corresponding to different ordinates of the calibration curve. Although these unknowns are not sufficient to calculate the partial constants, they enable us to calculate $\nu(\omega)$ and also $H(\omega)$, provided that $\frac{G i_{p}}{M}$ is known. In fact, from the expression resulting for $f$ when $s=e^{j \omega t}$, it is easily obtained

$$
H(\omega) e^{j \nu(\omega)}=\frac{j \sigma_{2} \omega}{\Phi(j \omega)}
$$


and hereof

$$
\begin{aligned}
& H(w)=\frac{\sigma_{2} \omega}{\left[\left(\left(p_{1}-\omega^{2}\right)^{2}+4 a_{1}^{2} \omega^{2}\right)\left(\left(p_{2}-\omega^{2}\right)+4 a_{2}^{2} \omega^{2}\right)\right]^{1 / 2}} ; \quad \nu=\frac{\pi}{2}-\left(\nu_{1}+\nu_{t}\right) \\
& \sin \nu_{1}=\frac{2 \alpha_{1} \omega}{\left[\left(p_{1}-\omega^{2}\right)^{2}+4 a_{1}^{2} \omega^{2}\right]^{1 / 2}} ; \quad \cos \nu_{1}=\frac{p_{1}-\omega^{2}}{\left[\left(p_{1}-\omega^{2}\right)^{2}+4 a_{1}^{2} \omega^{2}\right]^{1 / 2}} \\
& \sin \nu_{2}=\frac{2 \alpha_{2} \omega}{\left[\left(p_{2}-\omega^{2}\right)^{2}+4 a_{2}^{2} \omega^{2}\right]^{1 / 2}} ; \quad \cos \nu_{2}=\frac{p_{2}-\omega^{2}}{\left[\left(p_{2}-\omega^{2}\right)^{2}+4 a_{2}^{2} \omega^{2}\right]^{1 / 2}} .
\end{aligned}
$$

It is seen that $\nu$ is thoroughly determined from $a_{1}, a_{2}, p_{1}, p_{2}$, whereas $H(\omega)$ still needs the factor $\sigma_{2}$ to be known, and this is obtained from

$$
\sigma_{2}=\frac{K M}{\iota_{p} G}
$$

It is worthwhile to note that eliminating $x$ from equations (2) and since $s$ and $\dot{s}$ are causal functions, we get

$$
\dot{s}+2 \alpha s+\beta \dot{s}+2 \gamma s+\delta \int_{0}^{t} s d t=-\sigma_{2} f
$$

TABLE 3

Ordinates of a Theoretical Calibration Curve

\begin{tabular}{rccccccc}
\hline$t$ (sec) & $y_{n m m}$ & $t$ (sec) & $y_{m m}$ & $t(\mathrm{sec})$ & $y_{m m}$ & $t(\mathrm{sec})$ & $y_{m m}$ \\
\hline 00 & 0.0 & 28.0 & 74.937 & 560 & 33.042 & 84.0 & 9239 \\
40 & 8.529 & 32.0 & 70.812 & 600 & 27.985 & 88.0 & 7581 \\
80 & 29.540 & 360 & 65.020 & 64.0 & 23.546 & 920 & 6.204 \\
12.0 & 50.125 & 400 & 58.411 & 680 & 19.969 & 96.0 & 5.064 \\
160 & 65.033 & 44.0 & 51.585 & 720 & 16.400 & 1000 & 4124 \\
20.0 & 73.440 & 480 & 44.937 & 76.0 & 13.595 & 104.0 & 3.352 \\
24.0 & 76269 & 520 & 38.712 & 800 & 11.226 & 1080 & 2.720 \\
\hline
\end{tabular}

$\alpha, \beta, \gamma$, and $\delta$ are the second members of (7) and they can be obviously calculated from the first members. Thus, it is seen again that $f$ is fully determined when $\sigma_{2}$ and the four equivalent constants are known.

\section{DETERMINATION OF THE UNKNOWNS}

In contrast to the method based on the Fourier transform where a great number of ordinates of the calibration curve are required, the unknowns $a_{1}, a_{2}, p_{1}, p_{2}$, and $K$ can be obtained only from five of them by means of the closed expression (8). However, it is convenient to use many more and to compensate them by the leastsquares method. This can be based on the Newton-Raphson method, but since it sometimes becomes divergent, it is advisable to use it with some attenuation devices. To find suitable procedures, we tried: (a) the attenuated least-squares method suggested by Levenberg (1944), slightly modified, and (b) a method which may be called the method of attenuated steps which consists in multiplying the steps by a factor less than 1 .

The procedure (a) was tested multiplying by a factor of only the diagonal terms 
of the coefficient matrix, and also multiplying by a factor all the terms of the matrix. The procedure (b) was tested combining and not combining it with procedure (a). When combining with procedure (a), all the terms of the coefficient matrix were multiplied. The results obtained are shown in Table 4 where $R$ is the step attenuation factor, $Q$ is the factor applied to the terms of the coefficient matrix, $G$ is given by

$$
G=\sum(s-y)^{2}
$$

$y$ being the ordinates taken from the calibration curve, and

$$
D=\left[\left(\frac{\partial G}{\partial a_{1}}\right)^{2}+\left(\frac{\partial G}{\partial a_{2}}\right)^{2}+\left(\frac{\partial G}{\partial p_{1}}\right)^{2}+\left(\frac{\partial G}{\partial p_{2}}\right)^{2}+\left(\frac{\partial G}{\partial K}\right)^{2}\right]^{1 / 2}
$$

TABLE 4

Newton-Raphson Iterations With and without ATtenuation

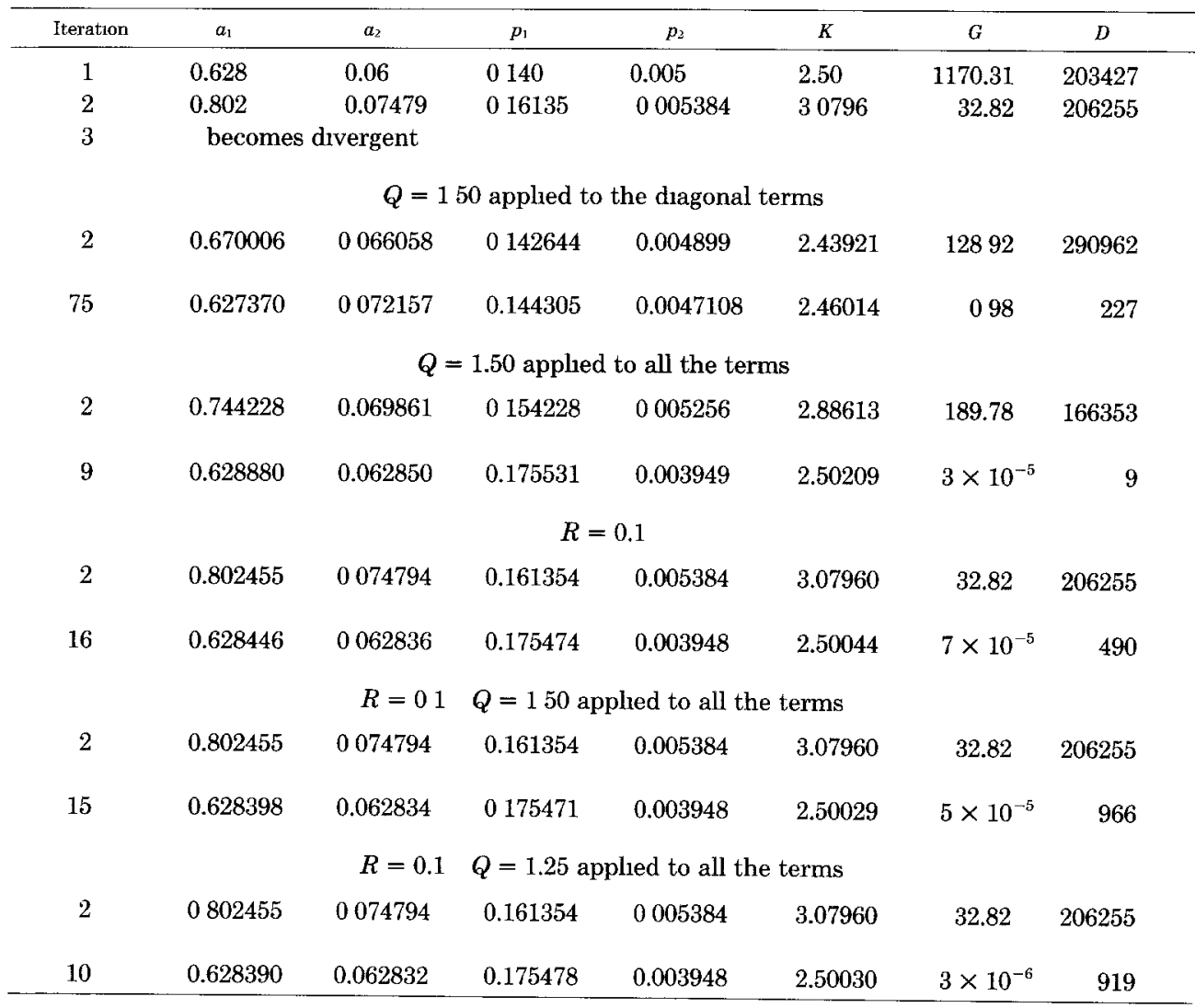

The solution is rigorously obtained when $D=0$, but it suffices that $D$ be less than a reasonable tolerance $\tau$. put

Being $\Delta Z$, the approximation to the solution, and $\epsilon_{G}$, the inaccuracy of $G$, we may

$$
\tau<\frac{\epsilon_{G}}{\Delta Z}
$$


Since

$$
\epsilon_{G}=2 \mu_{g} \sqrt{G}<2 \mu_{g} \delta \sqrt{N}
$$

where $N$ is the number of ordinates, $\delta$, their probable error, and $\mu_{g}$, the presumable least value of $s-y$, we may assume that $\epsilon_{G}=2 \mu_{g} \delta \sqrt{N}$.

Levenberg proposed to use a factor $Q$ which is variable with every iteration. As it may become too big not allowing the iteration process to proceed, we used instead a constant value between 1 and 2 . This factor and $R$ were used in procedure (b) only where $G$ increased.

The values $y$ used in our test are given in Table 3 . They were obtained from (8) putting $a_{1}=0.628319, a_{2}=0.0628319, p_{1}=0.175459, p_{2}=0.00394784$. These are the values corresponding to case (b) of Table $1 . K$ was assumed to be 2.50 in order that the ordinates be similar to those ordinarily given by the calibration curve.

TABLE 5

\begin{tabular}{|c|c|c|c|c|c|c|c|}
\hline & \multicolumn{7}{|c|}{ Constraints } \\
\hline & \multicolumn{3}{|c|}{ Case 1} & \multicolumn{4}{|c|}{ Case 2} \\
\hline & \multicolumn{3}{|c|}{$\begin{array}{l}n_{1}=0.418879 ; T_{1}=15.0 \mathrm{sec} \\
n_{2}=0.062832 ; T_{2}=100.0 \mathrm{sec} \\
\epsilon_{2}=0062832, h_{2}=100\end{array}$} & & \multicolumn{2}{|c|}{$\begin{array}{l}n_{1}=0418879 ; T_{1}=150 \mathrm{sec} \\
\epsilon_{1}=0.628319 ; h_{1}=15\end{array}$} & \\
\hline \multirow{3}{*}{$\begin{array}{c}\text { Un- } \\
\text { knowns }\end{array}$} & \multirow{3}{*}{ Expected } & \multicolumn{6}{|c|}{ Obtained } \\
\hline & & \multicolumn{3}{|c|}{$s_{2}$ case b from Table 2} & \multicolumn{3}{|c|}{$y$ from Table 3} \\
\hline & & No constraints & Case 1 & Case 2 & No constraints & Case 1 & Case 2 \\
\hline$a_{1}$ & 0.628319 & 0.454657 & 0598277 & 0.628715 & 0628446 & 0.627626 & 0628218 \\
\hline$a_{2}$ & 0.062832 & 0054023 & 0061991 & 0.062832 & 0062836 & 0.062812 & 0062832 \\
\hline$p_{1}$ & 0.175459 & 0.236426 & 0.178239 & 0176868 & 0175474 & 0.175350 & 0.175459 \\
\hline$p_{2}$ & 0.003948 & 0.003150 & 0003882 & 0.004062 & 0003948 & 0.00394628 & 0.003948 \\
\hline$K$ & $481,2.50$ & 535232 & 4.81112 & 4.99997 & 250044 & 2.49744 & 2.50 \\
\hline$\epsilon_{1}$ & - & - & 0597435 & - & - & 0.627606 & - \\
\hline$\epsilon_{2}$ & - & - & - & 00632287 & - & - & 0062832 \\
\hline$n_{2}$ & - & - & - & 0063993 & 一 & - & 0.062832 \\
\hline$T_{1}^{\prime}$ & 15.00 & 12.92 & 14.88 & 1494 & 15.00 & 15.00 & - \\
\hline$T_{2}^{\prime}$ & 100.00 & 11195 & 10084 & 9858 & 10000 & 100.02 & - \\
\hline$h_{1}^{\prime}$ & 1.50 & 094 & 1.42 & 149 & 1.50 & 1.50 & - \\
\hline$h_{2}^{\prime}$ & 1.00 & 096 & 0.99 & 0.99 & 1.00 & 1.00 & - \\
\hline$G$ & - & 1.73 & 179 & 54.8 & 000007 & 0.00001 & 0.02 \\
\hline$D$ & - & 1.04 & 035 & 2.07 & 490 & 0.05 & - \\
\hline
\end{tabular}

To choose a value for $\tau$, we assumed $\Delta Z=5 \times 10^{-7}, \mu_{g}=0.1$, and $\delta=0.0005$. Consequently, we adopted $\tau=200 \sqrt{N}$, and starting from certain approximate values, we expected to obtain the above values for the unknowns.

From Table 4, it is seen that using the Newton-Raphson method without attenuations, it may become impossible to arrive at a solution; however, Levenberg's modified method and the step attenuation method, can lead to it. It is also seen that the solution differs very little from what was expected and that convergency is better when not only the diagonal terms of the coefficient matrix are multiplied by $Q$, but all of them. 


\section{DETERMINATION UNDER CONSTRAINTS}

The long-period WWSSN instruments allow $n_{2}, \epsilon_{2}$, and $n_{1}$ to be determined quite well separately or to be left adjusted to some standard values of these partial constants; in fact, this is done by the stations from time to time. Therefore, one might wish to obtain the unknowns from the calibration curve under the constraints that some of the values of the partial constants are those assigned to them by the station. Taking this into account, we may consider the following cases

\begin{tabular}{|c|c|c|c|}
\hline Case & \multicolumn{3}{|c|}{ Known Constants } \\
\hline 1 & $n_{1}$ & $n_{2}$ & $\epsilon_{2}$ \\
\hline 2 & & $n_{1}$ & $\epsilon_{1}$ \\
\hline 3 & & $n_{2}$ & $\epsilon_{2}$ \\
\hline 4 & & $n_{1}$ & $n_{2}$ \\
\hline
\end{tabular}

In all these cases, the constraints are defined by equation (7) from which a dependence of some unknowns from the remaining can be obtained.

In case 1 , we may calculate $a_{2}, p_{2}$, and $\epsilon_{2}$ from $a_{1}$ and $p_{1}$ as follows

$$
p_{2}=\frac{n_{1}{ }^{2} n_{2}{ }^{2}}{p_{1}} ; \quad a_{2}=\frac{\epsilon_{2}\left(n_{2}{ }^{2}-n_{1}{ }^{2}\right)+a_{1}\left(p_{2}-n_{2}{ }^{2}\right)}{n_{2}{ }^{2}-p_{1}} ;
$$

$$
\epsilon_{1}=\frac{a_{1}\left(p_{2}-p_{1}\right)+\epsilon_{2}\left(p_{1}-n_{1}^{2}\right)}{n_{2}^{2}-p_{1}}
$$

and $a_{1}, p_{1}$, and $K$ should be obtained from the calibration curve. In case 2 we have

$$
n_{2}^{2}=\frac{p_{1} p_{2}}{n_{1}^{2}} ; \quad a_{1}=\frac{\epsilon_{1}\left(n_{1}^{2}-n_{2}^{2}\right)+a_{2}\left(p_{1}-n_{1}^{2}\right)}{n_{1}{ }^{2}-p_{2}} ; \quad \epsilon_{2}=\frac{a_{2}\left(p_{1}-p_{2}\right)+\epsilon_{1}\left(p_{2}-n_{2}{ }^{2}\right)}{n_{1}{ }^{2}-p_{2}}
$$

and $a_{2}, p_{1}, p_{2}$, and $K$ are obtained from the calibration curve. The same is valid for case 3; one must only interchange the subscripts 1 and 2.

Case 1 finds application particularly when one is interested in knowing $\epsilon_{1}$ whose determination is generally not easy. Case 2 occurs when trouble affects the galvanometer changing its period and damping, a fact that happens frequently due to some fungus developed in moisture existing in its container. Finally, in case 4, we put for $p_{2}$ the expression written in (12) and the unknowns to be obtained are $a_{1}, a_{2}$, $p_{1}$, and $K$.

Table 5 compares results obtained under different constraints and others free of them corresponding to the same readings. In it we also give the following additional values

$$
T_{1}^{\prime}=\frac{2 \pi}{\sqrt{p_{1}}} ; \quad T_{2}^{\prime}=\frac{2 \pi}{\sqrt{p_{2}}} ; \quad h_{1}^{\prime}=\frac{a_{1}}{\sqrt{p_{1}}} ; \quad h_{2}^{\prime}=\frac{a_{2}}{\sqrt{p_{2}}}
$$

which generally should differ little from $T_{1}, T_{2}, h_{1}$, and $h_{2}$, respectively.

The accuracy of the results is highly dependent on the accuracy to which the readings, $y$, on the calibration curve are made. This is very low before its maximum; it is, therefore, convenient to use the ordinates from this point onwards. 


\section{CoNCLUSIONS}

From the considerations made, the following conclusions have resulted.

1. The known fact that the release and the step current test produce the same calibration curve is theoretically explained.

2. Its explicit expression can easily be obtained by the method of the characteristic equation.

3. The seismograph frequency response is determined by the equivalent constants when a factor $K$, the reduced motor constant and the calibration current are known, and there is no need to know explicitly the values of the partial instrumental constants.

4. $K$ and the equivalent constants can be obtained from the calibration curve either freely or under the constraint that some of the partial constants be the ones given by the recording station.

5. Determinations under constraint are to be made when one wishes to obtain the seismometer damping coefficient as well as the equivalent constants when the galvanometer period and damping have been affected by some trouble.

6. The Newton-Raphson iteration method can be used to obtain the unknowns, but since it fails sometimes, becoming divergent, it is convenient to improve it using either the step attenuation procedure or Levenberg's attenuated leastsquares procedure modified by putting a reasonable constant instead of his variable factor and applying it to all the terms of the coefficient matrix.

\section{ACKNOWLEDGMENT}

The authors are indebted to the Centro Regıonal de Sismología para Amerıca del Sur (CERESIS) for making the publication of the present paper possible.

\section{REFERENCES}

Chakrabarty, S. K. and S. N. R. Chudhury. (1964). Response characteristıcs of electromagnetic seismographs, Bull. Selsm. Soc. Am. 54, 1455-1458.

Coulomb, J. and G. Grenet (1935). Nouveau pruncipes de construction des seismographes electromagnetiques, Ann. Phys. 3, 321-368.

Espinosa, A. F., G. H. Sutton, and H. J. Miller (1965). A transient technique for seismograph calibration, Institute of Science and Technology, Unversity of Michıgan.

Hagiwara, T. (1958). A note on the theory of electromagnetic seismograph, Bull. Earthquake Res. Inst., Tokyo Univ 36, 139-164.

Jarosch, H. and A. R Curtis (1973) A note on the calıbration of the electromagnetic se1smograph, Bull. Seism. Soc. Am. 63, 1145-1155.

Levenberg, H. (1944). A method for the solution of certam non-linear problems in least squares, $Q$ Appl Math 2, 164-169.

Mitchell, B and M. Landisman (1969) Electromagnetic seismograph constants by least square inversion, Bull. Sersm. Soc. Am. 59, 1335-1348

Morencos, T. J (1972) Respuesta teónca de sistema sismometrico galvanometro, revista de la real academa de ciencias exactas, Flsucas y Naturales de Madrid X, 147-180.

Salvill, R A., E. W. Carpenter, and J. K. Wright (1962) The derivation and solution of indicator equations for seismometer galvanometer combination including the effect of seismometer inductance, Geophys. J. 6, 409-425.

Teng, T. L. and A. Ben Menahem (1965). Mechanısm of deep earthquakes from spectra of isolated body waves signals, Geophys. J. Res. 70, 5157-5170.

Tobyáš, V and Z. Hubernova (1969) Equivalent and partıal constants of electromagnetic seismographs. Geophysicalnı Sbornık XVI, 79-113

Universidad Nacional de la Plata

Observatorio Astronomico

1900, La Plata, Argentina

Manuscript received October 14, 1977 